\title{
Lipoma de la cavidad oral. Descripción de casos.
}

\author{
Lipoma of the oral cavity. Cases description.
}

\author{
Enrique Darío Amarillas Escobar*
}

RESUMEN

El lipoma es un tumor benigno derivado de tejido adiposo maduro que se presenta con poca frecuencia en la cavidad oral, pero de interés para el clínico estomatológico por su distintiva presentación en esta región. A continuación se reportan dos casos clínicos de lipoma simple localizados en la mucosa vestibular y piso de boca que fueron tratados mediante eliminación quirúrgica.

Palabras clave: Lipoma, lipoma bucal, tumor oral benigno.
ABSTRACT

Lipoma is a benign neoplasm derived of mature adipose tissue that occurs infrequently in the oral cavity, but of interest to the stomatologic clinician for its distinctive presentation in this region. Next, two cases of simple lipoma located in the vestibular mucosa and floor of the mouth that were treated by surgical excision are presented.

Keywords: Lipoma, oral lipoma, oral benign tumors.

\section{INTRODUCCIÓN}

$\mathrm{A}$ ddison y Gall fueron los primeros en reportar un lipoma en 1851; sin embargo, Dequeker y colaboradores en su descripción de la célebre obra Mona Lisa pintada por Leonardo da Vinci en el siglo XVI sugieren la presencia de un lipoma en el dorso de la mano derecha, lo que podría indicar que esta lesión ya era conocida mucho antes. ${ }^{1}$

Como antecedente estomatológico, el lipoma bucal fue descrito inicialmente por Roux en 1848 como «épulis amarillo» en una revisión de casos que mostraban masas alveolares. $^{2}$

El lipoma es un tumor benigno de origen mesenquimatoso compuesto por adipocitos maduros que no presentan atipias. Su frecuencia de aparición en la región de cabeza y cuello corresponde a $15-20 \%$ de los casos, ${ }^{3}$ siendo los sitios intrabucales más comúnmente afectados por su mayor contenido de tejido adiposo, la mucosa y el surco vestibular con $50 \%$ de los casos, seguidos de la lengua, piso de boca, labios, paladar y región retromolar como sitios menos comunes, ${ }^{4}$ y de forma extrabucal puede aparecer en la región parotídea y submandibular. ${ }^{5}$

El lipoma bucal corresponde a $1-4 \%$ de todos los tumores benignos de esta región. ${ }^{3}$ Clínicamente se manifiesta como una lesión asintomática, de crecimiento lento, de forma redondeada u ovoide, bien delimitada, de consistencia blanda, sésil o pediculada, libremente desplazable y cubierta por mucosa de características normales donde los vasos superficiales se pueden hacer evidentes. Se presenta generalmente como una lesión única, aunque puede aparecer en mayor número. Su típico color amarillo puede evidenciarse si la mucosa que lo cubre es delgada, siendo de esta manera más sencillo su diagnóstico; sin embargo, puede ser más complejo si la lesión presenta una localización profunda. ${ }^{4,6}$

\footnotetext{
* Cirujano Maxilofacial. Profesor de asignatura de la Licenciatura de Cirujano Dentista de la Facultad de Estudios Superiores Zaragoza de la Universidad Nacional Autónoma de México. Profesor asociado de la Licenciatura de Estomatología de la Universidad Autónoma Metropolitana Unidad Xochimilco.
}

Recibido: 25 de octubre de 2019. Aceptado: 26 de octubre de 2021.

Citar como: Amarillas EED. Lipoma de la cavidad oral. Descripción de casos. Rev ADM. 2021; 78 (6): 361-364. https://dx.doi.org/10.35366/102979

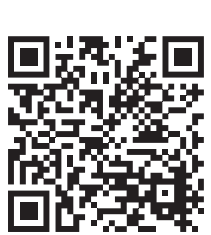


En la mayoría de los casos su tamaño oscila entre 1.5-2.5 cm de diámetro. ${ }^{3}$ Dependiendo de su dimensión y localización puede provocar incomodidad y dificultad para la masticación, deglución, fonación, respiración e higiene bucal. ${ }^{4}$

El tratamiento consiste en la escisión quirúrgica completa de la lesión para evitar así la recurrencia. ${ }^{3}$

\section{PRESENTACIÓN DE CASOS CLÍNICOS}

\section{Caso 1}

Paciente masculino de 57 años de edad, quien acude a la Clínica Multidisciplinaria de Atención a la Salud (CUAS) Estado de México de la Facultad de Estudios Superiores Zaragoza (FES-Z) de la Universidad Nacional Autónoma de México (UNAM) por presentar un aumento de volumen asintomático, de cinco años de evolución y de crecimiento lento y progresivo, cuya dimensión le causaba dificultades para la alimentación y fonación. Sin antecedentes médicos y dentales contribuyentes. Tras la exploración física intrabucal se observó un aumento de volumen en la mucosa vestibular del lado derecho cercana a la comisura labial y al surco vestibular inferior, redondeado, bien delimitado, de consistencia blanda, base sésil y libremente móvil. La mucosa suprayacente sin alteraciones, con finos vasos sanguíneos evidentes en la superficie (Figura 1). No refirió antecedentes de descarga purulenta, sangrado o trauma. Los estudios de laboratorio prequirúrgicos de rutina del paciente con valores de referencia dentro de los parámetros normales.

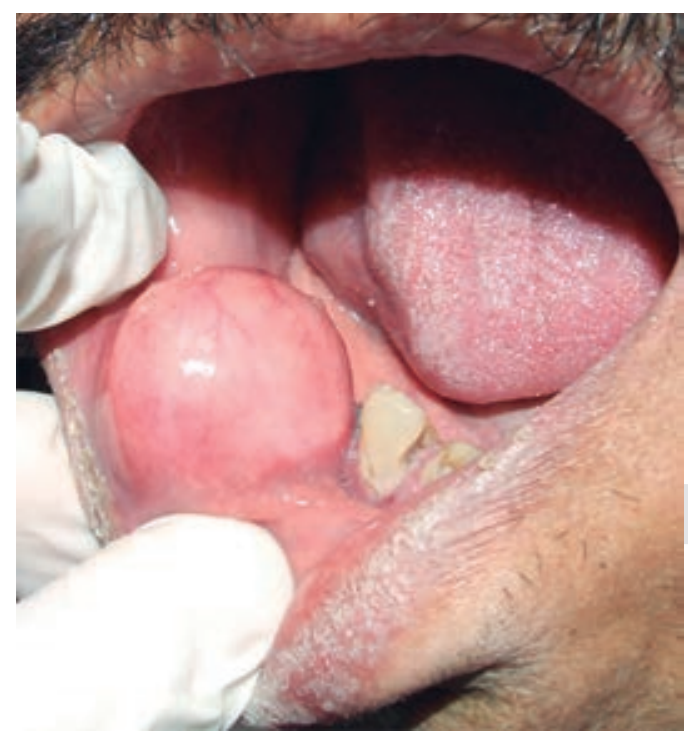

Figura 1: Presentación clínica de la patología bucal.

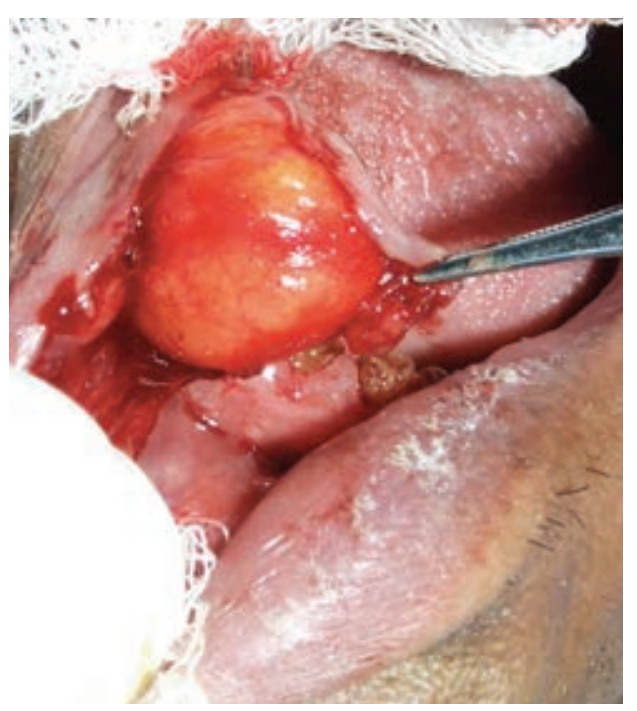

Figura 2: Abordaje quirúrgico.

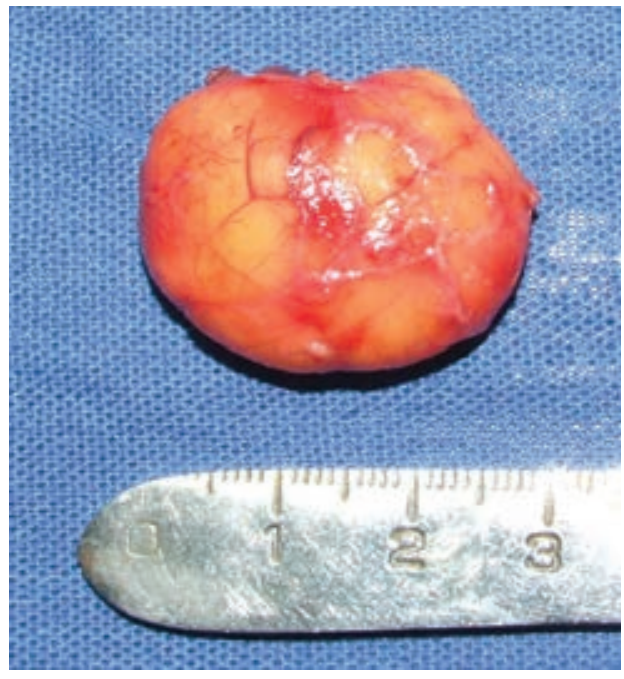

Figura 3: Espécimen quirúrgico obtenido.

Se estableció un diagnóstico presuntivo de lipoma bucal y se realizó su eliminación quirúrgica bajo anestesia local, prestando particular atención en el cuidado del paquete neurovascular mentoniano por su cercanía con la lesión (Figura 2). El espécimen quirúrgico obtenido presentó una dimensión de $3 \mathrm{~cm}$ en su eje mayor y fue enviado a la Unidad de Patología Bucal de la FES-Z, cuya evaluación histopatológica confirmó el diagnóstico inicial (Figura 3). Después de seis años de seguimiento y hasta el momento, la paciente no ha mostrado datos de recurrencia. 


\section{Caso 2}

Paciente femenino de 69 años de edad, aparentemente sana, sin antecedentes de importancia para el padecimiento actual. Posterior al interrogatorio y exploración física, se destacó un aumento de volumen en el piso de boca del lado derecho, asintomático, de un año de evolución, sin causa aparente de formación, de consistencia blanda, sésil, móvil, que desplazaba el frenillo lingual hacia el lado contrario. La mucosa suprayacente a la lesión sin cambios, con vasos delgados en su superficie (Figura 4). Los conductos salivales submandibulares se encontraron permeables. Sin adenomegalias. No se encontraron datos

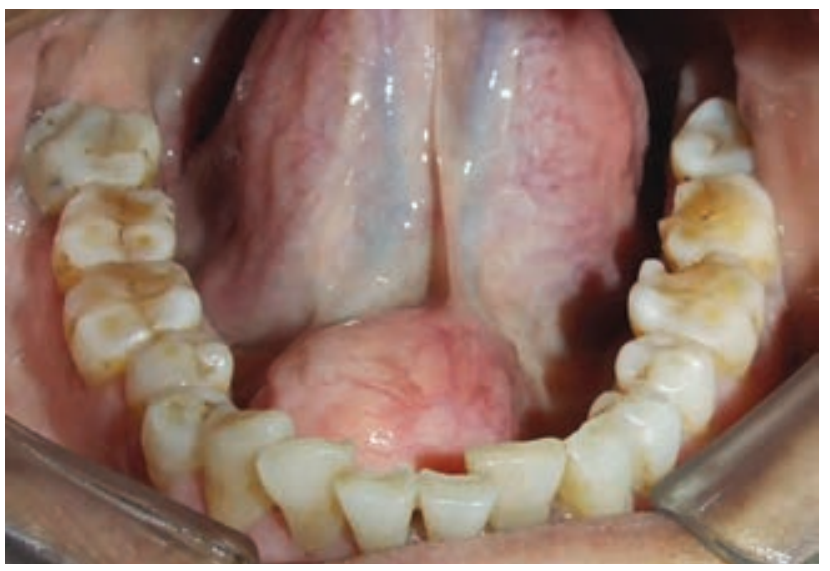

Figura 4: Localización del tumor bucal.

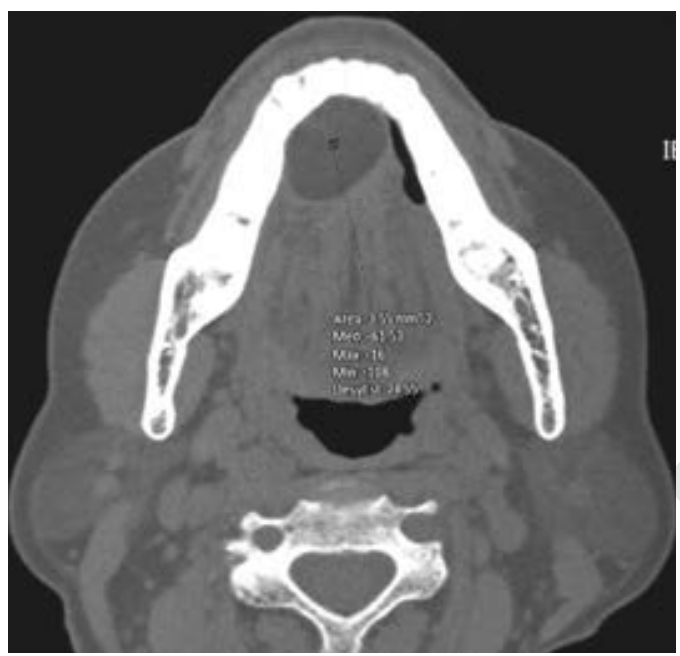

Figura 5: Corte tomográfico que muestra la imagen correspondiente con la lesión bucal.

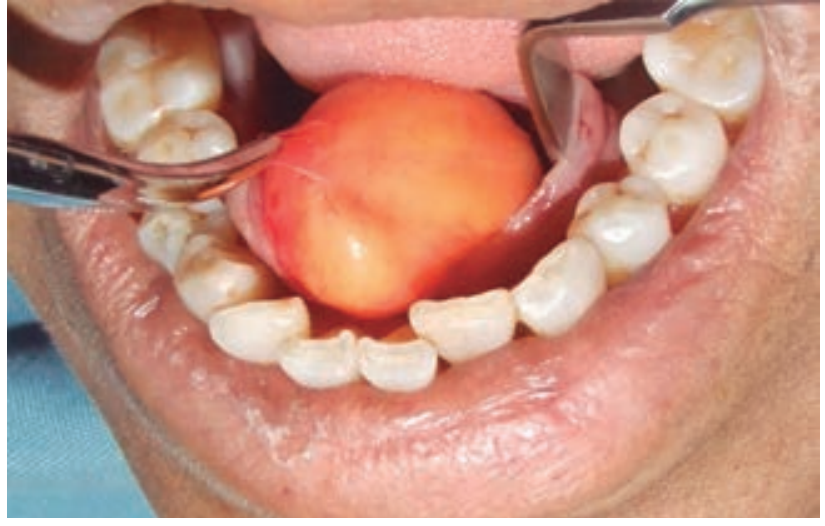

Figura 6: Detalle del momento transoperatorio.

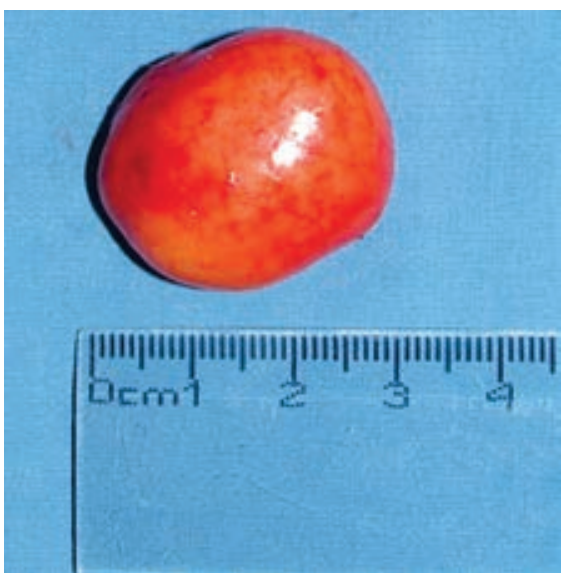

Figura 7: Pieza quirúrgica extirpada.

de infección o etiología por patología dentaria. Se realizó una punción y aspiración diagnóstica no obteniendo material. Se hizo una tomografía computarizada simple donde se evidenció una imagen unilocular, bien circunscrita, con contenido heterogéneo, cuyas densidades se encontraban entre -80 y $-120 \mathrm{uH}$ (unidad Hounsfield), sin alteraciones de la musculatura y demás estructuras anatómicas adyacentes (Figura 5). Considerando la apariencia benigna de la lesión y estableciendo un diagnóstico presuntivo de quiste dermoide versus lipoma, se procedió a su eliminación quirúrgica bajo anestesia locorregional vía intrabucal. Una vez obtenido el espécimen quirúrgico, se consideró sólo el diagnóstico presuntivo de lipoma por su característico color amarillo (Figura 6). El reporte histopatológico confirmó el diagnóstico clínico. El tumor presentó una dimensión de $3 \times 2.5 \times 1.5 \mathrm{~cm}$ (Figura 7). Durante un curso de seis años, la paciente no ha mostrado 
ninguna complicación derivada de la patología o de la intervención quirúrgica.

\section{DISCUSIÓN}

El lipoma es el tumor de los tejidos blandos más frecuente del organismo humano. Puede aparecer en cualquier sitio del cuerpo donde exista tejido adiposo, siendo habitual en la espalda alta, hombros y región proximal de las extremidades y menos común en la cavidad bucal donde representa sólo $2.2 \%$ de los casos. ${ }^{4,7}$ Se puede presentar en cualquier edad, pero es más común en adultos de 40-60 años, con predominio en hombres (1.5:1). ${ }^{4}$ Se ha reportado también casos congénitos. ${ }^{8}$

Comúnmente, el lipoma aparece de manera subcutánea y submucosa, pero también puede desarrollarse entre las fibras musculares donde se le ha denominado lipoma intramuscular o infiltrante ${ }^{9}$ sobre la superficie ósea ${ }^{10} \mathrm{o}$ de forma intraósea, ${ }^{11}$ o involucrar el tejido nervioso, sinovial ${ }^{12}$ y las glándulas salivales. ${ }^{9}$

Se desconoce la etiología del lipoma, pero se han descrito como posibles factores causales la herencia, degeneración grasa, alteraciones hormonales, trauma, infección, infarto, irritación crónica, metaplasia de músculo y nidos de células embrionarias lipoblásticas. ${ }^{3}$

Como auxiliares de diagnóstico suelen utilizarse la ultrasonografía, la tomografía computarizada y la resonancia magnética que evidenciarán imágenes correspondientes con tejido blando de naturaleza grasa. ${ }^{12}$

El lipoma está compuesto histológicamente por tejido adiposo, dividido en lóbulos por septos de tejido conectivo fibroso y rodeado por una delgada cápsula. En pocos casos la cápsula está ausente. Microscópicamente no es posible distinguir entre un lipoma y el tejido adiposo normal; sin embargo, el metabolismo del lipoma es diferente, ya que la grasa que contiene no es utilizada para la producción de energía durante la inanición. ${ }^{13}$

Además del lipoma simple, que es el tipo más común, se ha reportado también una serie de variantes histopatológicas de presentación en la cavidad bucal como el fibrolipoma, mixolipoma, angiolipoma, lipoma pleomórfico/fusocelular, osteolipoma y condrolipoma. 5, , 10,14-17

Las complicaciones no son frecuentes, pero las lesiones de largo tiempo de evolución pueden transformarse en un liposarcoma. ${ }^{4}$

\section{CONCLUSIONES}

Como se ha explicado ya, el lipoma es una patología que se presenta con poca frecuencia en la cavidad oral, pero no por este hecho debe ser desconocida por el clínico, más bien, se debe considerar como uno de los diagnósticos diferenciales durante la evaluación de las lesiones tumorales de la cavidad oral.

\section{REFERENCIAS}

1. Dequeker J, Muls E, Leenders K. Xanthelasma and lipoma in Leonardo da Vinci's Mona Lisa. Isr Med Assoc J. 2004; 6 (8): 505-506.

2. Pereira T, Shetty S, Sapdhare S, Tamgadge A. Oral fibrolipoma: a rare histological variant. Indian J Dent Res. 2014; 25 (5): 672-674.

3. Coelho RCP, Oliveira EM, Silva GCC, Aguiar EG, Moreira AN, Souza LN. Intraoral excision of a huge cheek lipoma. J Craniofac Surg. 2018; 29 (1): e96-e97.

4. Devi AN, Sowbhagya MB, Balaji P, Mahesh Kumar TS. An uncommon case of fibrolipoma. Indian J Dent Res. 2017; 28 (6): 699-701.

5. Furlong MA, Fanburg-Smith JC, Childers EL. Lipoma of the oral and maxillofacial region: site and subclassification of 125 cases. Oral Surg Oral Med Oral Pathol Oral Radiol Endod. 2004; 98 (4): 441-450.

6. Trento GDS, Stringhini DJ, Rebellato NLB, Scariot R. Extra-oral excision of a buccal fat pad lipoma. J Craniofac Surg. 2017; 28 (3): e226-e227.

7. Studart-Soares EC, Costa FWG, Sousa FB, Alves APNN, Osterne RLV. Oral lipomas in a Brazilian population: a 10-year study and analysis of 450 cases reported in the literature. Med Oral Patol Oral Cir Bucal. 2010; 15 (5): e691-696.

8. Ohyama Y, Uzawa N, Yamashiro M, Yamaguchi S. Congenital lipoma of the hard palate: case report. Br J Oral Maxillofac Surg. 2017; 55 (6): 626-627.

9. Fregnani ER, Pires FR, Falzoni R, Lopes MA, Vargas PA. Lipomas of the oral cavity: clinical findings, histological classification and proliferative activity of 46 cases. Int J Oral Maxillofac Surg. 2003; 32 (1): 49-53.

10. Omonte SV, de Andrade BA, Leal RM, Capistrano HM, Souza PE, Horta MC. Osteolipoma: a rare tumor in the oral cavity. Oral Surg Oral Med Oral Pathol Oral Radiol. 2016; 122 (1): e8-e13.

11. Lee KM, Kim HG, Lee YH, Kim EJ. mDixon-based texture analysis of an intraosseous lipoma: a case report and current review for the dental clinician. Oral Surg Oral Med Oral Pathol Oral Radiol. 2018; 125 (3): e67-e71.

12. Choi HJ, Byeon JY. Symptomatic intraoral submuscular lipoma located nearby mental foramen. J Craniofac Surg. 2016; 27 (5): e457-459.

13. Epivatianos A, Markopoulos AK, Papanayotou P. Benign tumors of adipose tissue of the oral cavity: a clinicopathologic study of 13 cases. J Oral Maxillofac Surg. 2000; 58 (10): 1113-1117.

14. Vecchio G, Amico P, Caltabiano R, Colella G, Lanzafame S, Magro G. Spindle cell/pleomorphic lipoma of the oral cavity. J Craniofac Surg. 2009; 20 (6): 1992-1994.

15. Villarroel Dorrego M, Papp Y, Shelley MJ, Barrett AW. Chondroid lipoma of the tongue: a report of two cases. Oral Maxillofac Surg. 2014; 18 (2): 219-222.

16. Juliasse LW, Nonaka CF, Pinto LP, Freitas Rde A, Miguel MC. Lipomas of the oral cavity: clinical and histopathologic study of 41 cases in a Brazilian population. Eur Arch Otorhinolaryngol. 2010; 267 (3): 459-465.

17. De Freitas MA, Freitas VS, de Lima AA, Pereira FB Jr, dos Santos JN. Intraoral lipomas: a study of 26 cases in a Brazilian population. Quintessence Int. 2009; 40 (1): 79-85.

\section{Correspondencia:}

Enrique Darío Amarillas Escobar

E-mail: dario_amarillas@hotmail.com 